\title{
XbarGen: a Memristor Based Boolean Logic Synthesis tool
}

\author{
Marcello Traiola ${ }^{1}$, Mario Barbareschi ${ }^{1}$, Antonino Mazzeo ${ }^{1}$, Alberto Bosio ${ }^{2}$ \\ ${ }^{1}$ DIETI, University of Naples Federico II \\ ${ }^{2}$ LIRMM, Université Montpellier \\ Naples, Italy \\ m.traiola@studenti.unina.it \\ Montpellier, France \\ bosio@lirmm.fr \\ \{mario.barbareschi, mazzeo\}@unina.it
}

\begin{abstract}
The shrinking process of CMOS technology is reaching its physical limits, thus impacting on several aspects, such as performances, power consumption and many others. Alternative solutions are under investigation in order to overcome CMOS limitations. Among them, the memristor is one of the promising technologies. Several works have been proposed so far, describing how to implement boolean logic functions employing memristors in a crossbar architecture. In this paper, we propose a tool able to automatically map any boolean function to a memristor based crossbar implementation. The proposed tool helps to perform a design space exploration to identify the best implementation w.r.t. performances and area overhead.

Index terms-Memristor crossbar, Design Space Exploration, Boolean Functions. Circuit Synthesis
\end{abstract}

\section{INTRODUCTION}

Today's computing devices are based on the CMOS technology, that is the subject of the famous Moore's Law [1] predicting that the number of transistors in an integrated circuit will be doubled every two years. Despite the advantages of the technology shrinking, we are facing the physical limits of CMOS. Among the multiple challenges arising from technology nodes lower than $20 \mathrm{~nm}$, we can highlight the high leakage current (i.e., high static power consumption), reduced performance gain, reduced reliability, complex manufacturing process leading to low yield and complex testing process, and extremely costly masks [2], [3], [4], [5].

Additionally, the expected never-ending increasing of performances is indeed no longer true. Looking in more detail, the classical computer architectures, either von Neumann or Harvard, divide the computational element (i.e., CPU) from the storage element (i.e., memory). Therefore, data have to be transferred inside the computational element in order to be processed and then transferred back to be stored. The main problem of this paradigm is the bottleneck due to the data transfer time limited by the bandwidth. For example, transferring one TeraByte at the rate of $1 \mathrm{Gbit} /$ second requires more than two hours.

Many new technologies are under investigation, among them the memristor is a promising one [9]. The memristor is a non-volatile device able to act as both storage and information processing unit that presents many advantages: CMOS process compatibility, lower cost, zero standby power, nanosecond switching speed, great scalability, high density and non-volatile capability [10], [11]. Thanks to its nature (i.e., computational as well as storage element), the memristor is exploited in different kind of applications, such as neuromorphic systems [12], non-volatile memories [13], computing architecture for data-intensive applications [6].

A fundamental component of any kind of computing architecture is the implementation of boolean logic functions. In [14], the authors proposed a methodology for the synthesis of boolean logic function on a memristor crossbar. Their work showed that is possible to implement any kind of boolean function on a memristor crossbar. However, the experimental results have been carried out on a couple of small circuits only due to the lack of an automated synthesis tool. In this paper, we aim to extend the work of [14] by presenting a tool able to automatically map any boolean function to a memristor based crossbar implementation. Moreover, we investigate the impact of different synthesis optimization parameters on the memristor crossbar to evaluate area and performances.

The remainder of the paper is structured as following. Section II presents the state of the art and provides the required background on the memristor based computation. Section III details the proposed memristor and crossbar model as well as the synthesis methodology, while the Section IV gives the experimental results. Finally, the Section V draws the conclusions.

\section{BACKGROUND AND STATE OF THE ART}

In this section we provide the basics about the memristor modeling, as well as the way how the memristor can be exploited to implement a given boolean function.

\section{A. Memristor model}

A memristor is a non-linear electrical component whose electrical resistance is not constant but depends on the history of the charge flowed through the device itself. Since we intend to implement a digital circuit, we refer to the memristor Voltage-Current relation depicted in Figure 1, detailed in [8], 
as the best solution for modeling the memristor's behavior (i.e., thanks to the ideal response to a pulse-wave). Thus, as the Figure 1 shows, the voltage applied to the memriristor's terminals does not change its resistance until it crosses a threshold. In the adopted ideal model, the upper and the lower thresholds have the same absolute value.

We resort to the Snider Boolean Logic (SBL) [8] convention whereby a lower resistance (steeper curve denoted as $\mathrm{R}_{\mathrm{ON}}$ ) represents a logic ' 0 ' while an higher resistance (lower slope curve denoted as $\mathrm{R}_{\mathrm{OFF}}$ ) represents a logic ' 1 '.

Two basic operations can be performed, defined as SET and RESET. The first one allows to program the memristor to $\mathrm{R}_{\mathrm{ON}}$ and thus at logic ' 0 ', while the second one programs the memristor to $\mathrm{R}_{\mathrm{OFF}}$ that corresponds to logic ' 1 '. The Figure 1 depicts SET and RESET operations as described by Xie et al. in [14]

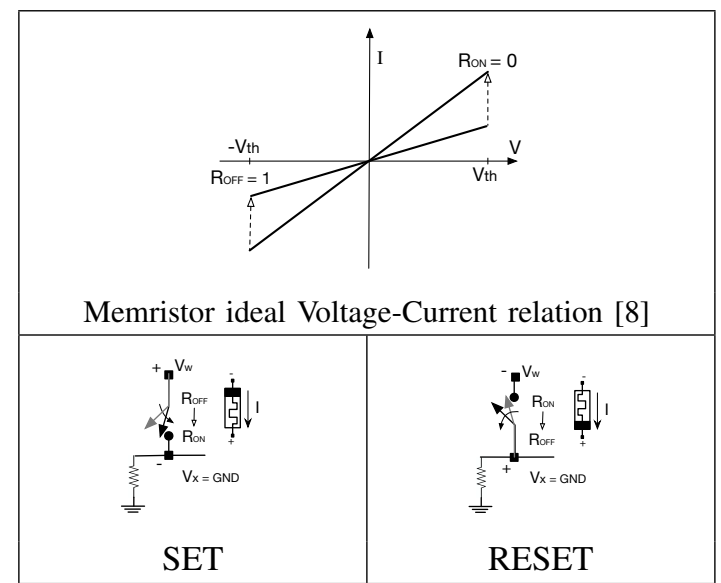

Figure 1. Set and Reset operations [14]

\section{B. Fast Boolean Logic Circuits}

Snider proposed in [8] a design methodology for memristor crossbar that aimed to implement boolean functions. This design was then improved by Xie et al. in [14]. Let us briefly recall their proposition referring to it as Fast Boolean Logic Circuit $(\boldsymbol{F B L C})$. First, the logic circuit requires that the Boolean function is expressed in the SOP format:

$$
f=M_{1}+M_{2}+\ldots+M_{n}=\underbrace{\overline{\overline{M_{1}} \cdot \overline{M_{2}} \cdots \cdots \overline{M_{n}}}}_{\text {NOT }}
$$

Then, as Figure 2-a shows, FBLC is divided in blocks, useful to accomplish FSM's steps (fig 2-b) which are:

INA: initialize all the memristors to $\mathrm{R}_{\mathrm{OFF}}$;

RI: $\quad$ the input block receives the inputs;

CFM configure all minterms simultaneously, in parallel;

EVM: $\quad$ evaluate all minterms simultaneously (NAND);

EVR: evaluate results: $\bar{f}$ is calculated (AND);

INR: invert results: $\bar{f}$ need to be inverted to achieve $f$ SO: $\quad$ send outputs: the result captured in OL is sent out.

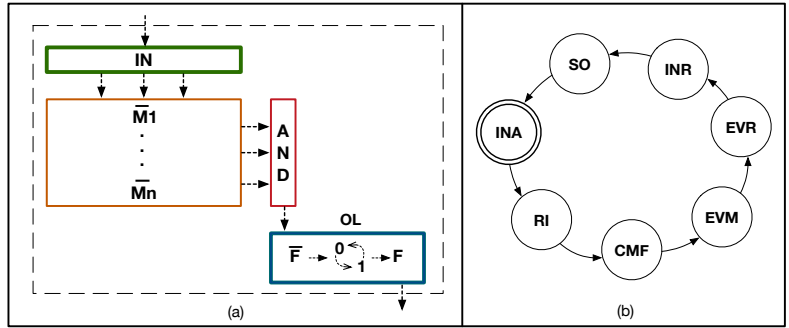

Figure 2. Fast Boolean Logic Circuit

Below, the description of the blocks:

- input block, where inputs are stored during the RI step;

- minterms block, where minterms are configured during CFM and evaluated during EVM;

- AND block, where results of EVM are stored and AND operation is performed during EVR;

- output block, where results of EVR are stored and inversion operation is performed during INR;

For the purpose of realizing each step of the FSM, the authors proposed some primitive operations that we summarize in Figure 3.

Each of these operations can be performed using as many input and output memristors as desired.

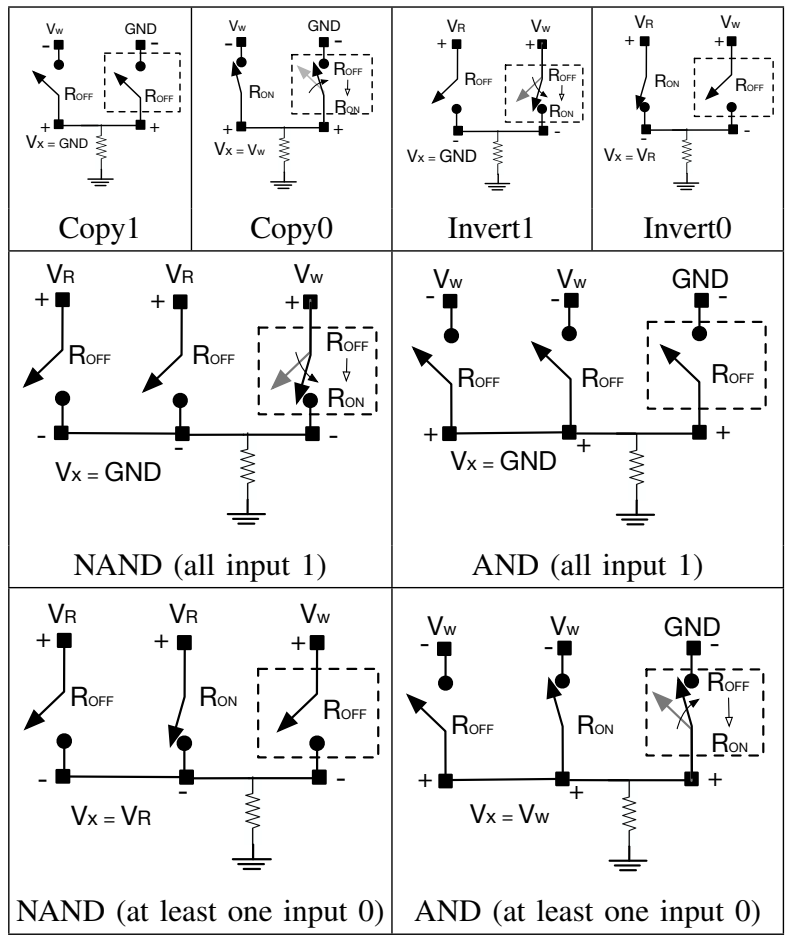

Figure 3. FBLC primitive operations

By driving the crossbar's nano-wires with the right voltages during each step, it is possible to calculate a boolean function in a constant number of steps. We report below in Figure 4 an example of crossbar for a simple 2 inputs / 1 output function, built following the FBLC approach. 


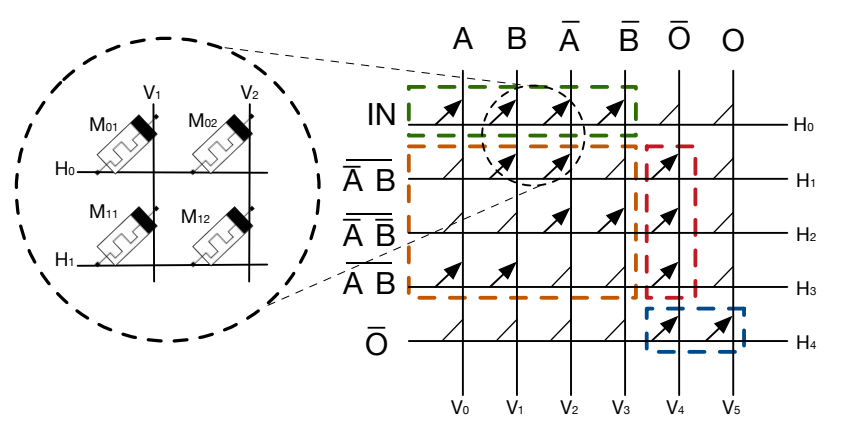

$$
O=A B+\bar{A} B+\overline{A B}=\overline{\overline{A B} \cdot \overline{\bar{A}} B \cdot \overline{\overline{A B}}}
$$

Figure 4. FBLC example

\section{TOWARDS AUTOMATIC CROSSBAR SYNTHESIS}

Due to the lack of an automated process of translation from a given boolean function to a memristor based crossbar architecture, deeply investigating about memristor based crossbar circuits turns out to be really hard to accomplish. Therefore, in order to overcome these problems, we developed XbarGen.

XbarGen is a command line tool written in $\mathrm{C}++$ which, starting from a boolean function described in the Synopsys equation format - EQN - (fig 5), executes the mapping to a memristor based crossbar architecture and produces a schematic view of the crossbar(s).

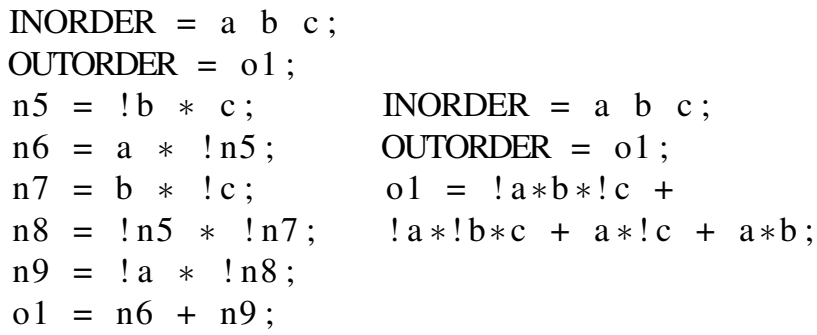

Figure 5. Synopsys equation format (EQN)

First, the tool operates an analysis of the given function. As the figure 5 shows, it is possible either that a function's output depends directly on its inputs either that it depends on "intermediate values", which indeed depends on inputs.

Thus, we will call level a set of inputs and outputs such that:

- inputs are independent from one another

- each output depends only on inputs

In this way we are able to describe a boolean function as a set of levels each one dependent from one another (figure 6)

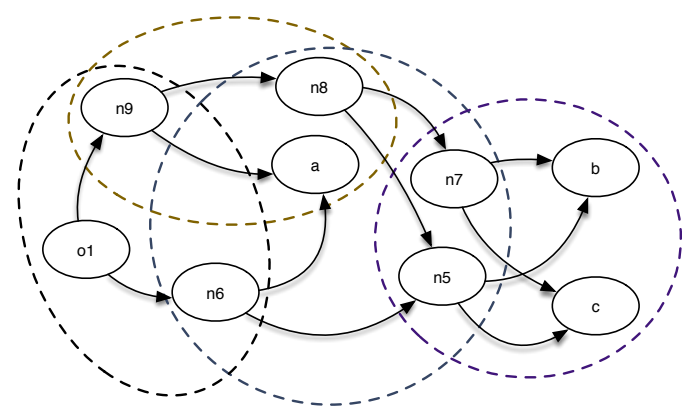

Figure 6. Function's levels

Second, the tool performs a mapping to one or more crossbars depending on how many levels it finds. Mapping to a crossbar means that inputs, outputs and related minterms of each level are translated into a FBLC, as explained in [14] and briefly reminded above. It is worth noting that, when more crossbars are produced, they must be connected together in series, as proposed by Snider in [8] and depicted in figure 7 . Consequently, the latency of the circuit grows proportionally to the number of serially connected crossbars.

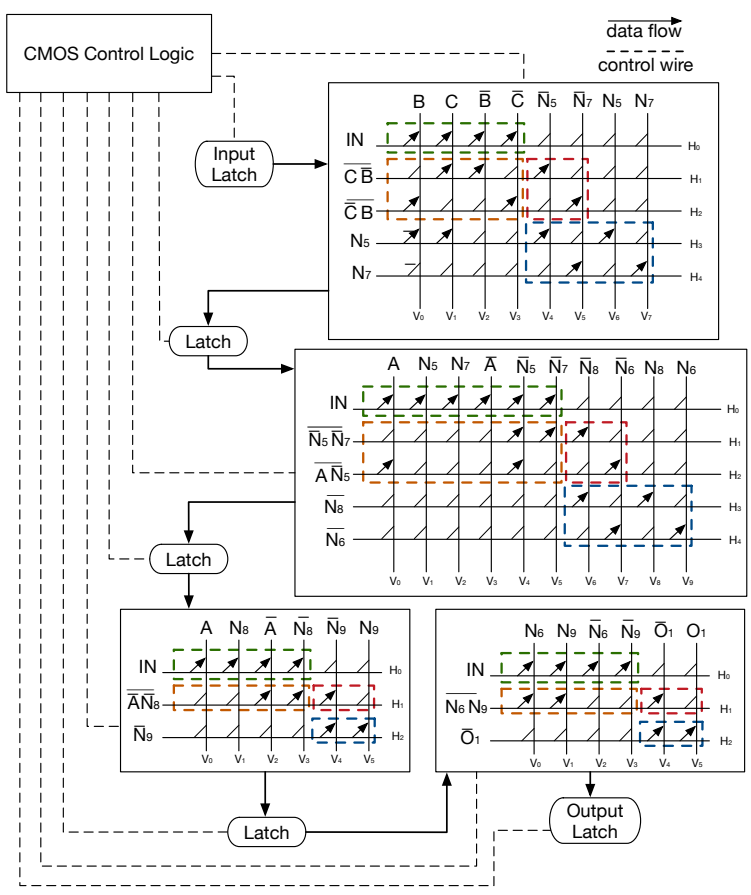

Figure 7. Snider Boolean Logic Circuit

\section{EXPERIMENTS AND RESULTS}

This section provides the experimental results achivied by the proposed synthesis tool and the adopted crossbar model. We also compare the proposed approach with the state of the art. 


\begin{tabular}{|c|c|c|c|c|c|c|c|c|c|c|c|c|c|c|c|c|c|c|c|c|c|c|c|}
\hline \multirow{2}{*}{ bmark } & \multirow{2}{*}{ Inputs } & \multicolumn{4}{|c|}{ Chakraborti et al. [7] } & \multicolumn{6}{|c|}{ Single Crossbar } & \multicolumn{6}{|c|}{ Multiple Crossbars } & \multicolumn{6}{|c|}{ Multiple Crossbars (optimized) } \\
\hline & & $M_{s}$ & $O P_{s}$ & $M_{p}$ & $O P_{p}$ & $N_{\min }$ & $N_{m e m}$ & A & $\mathrm{C}$ & $N_{C}$ & $T_{X b G}$ & $N_{\min }$ & $N_{m e m}$ & A & $\mathrm{C}$ & $N_{C}$ & $T_{X b G}$ & $N_{\min }$ & $N_{m e m}$ & \begin{tabular}{|l|} 
A \\
\end{tabular} & $\mathrm{C}$ & $N_{C}$ & $T_{X b G}$ \\
\hline rd53f1 & 5 & 7 & 58 & 15 & 34 & 6 & 48 & 96 & 7 & 1 & 4,40771 & 16 & 120 & 492 & 56 & 8 & 13,1694 & 11 & 87 & 378 & 35 & 5 & 9,26288 \\
\hline $\mathrm{rd} 53 \mathrm{f} 2$ & 5 & 8 & 59 & 17 & 35 & 20 & 132 & 264 & 7 & 1 & 5,71666 & 27 & 203 & 1318 & 49 & 7 & 17,8544 & 25 & 191 & 1108 & 49 & 7 & 17,5566 \\
\hline rd53f3 & 5 & 7 & 41 & 11 & 35 & 16 & 108 & 216 & 7 & 1 & 7,27523 & 22 & 156 & 894 & 42 & 6 & 15,5731 & 13 & 93 & 326 & 42 & 6 & 10,0649 \\
\hline xor5_d & 5 & 7 & 41 & 11 & 35 & 16 & 108 & 216 & 7 & 1 & 6,88398 & 22 & 156 & 894 & 42 & 6 & 14,4109 & 13 & 93 & 326 & 42 & 6 & 10,9131 \\
\hline con1f1 & 7 & 8 & 59 & 17 & 47 & 5 & 37 & 98 & 7 & 1 & 4,11653 & 13 & 105 & 338 & 49 & 7 & 17,004 & 9 & 69 & 242 & 28 & 4 & 9,66641 \\
\hline $\operatorname{con} 2 \mathrm{f} 2$ & 7 & 9 & 47 & 18 & 47 & 6 & 38 & 96 & 7 & 1 & 4,71909 & 16 & 122 & 490 & 49 & 7 & 18,7644 & 10 & 74 & 284 & 28 & 4 & 9,93731 \\
\hline rd73f1 & 7 & 8 & 84 & 18 & 48 & 42 & 330 & 704 & 7 & 1 & 10,9064 & 113 & 851 & 15232 & 98 & 14 & 92,7335 & 80 & 606 & 9354 & 70 & 10 & 69,9187 \\
\hline rd73f2 & 7 & 7 & 57 & 13 & 49 & 64 & 528 & 1056 & 7 & 1 & 14,0526 & 34 & 244 & 1842 & 56 & 8 & 25,8204 & 19 & 139 & 706 & 42 & 6 & 12,7191 \\
\hline rd73f3 & 7 & 7 & 106 & 25 & 46 & 35 & 275 & 592 & 7 & 1 & 9,78765 & 59 & 459 & 4186 & 91 & 13 & 39,1519 & 40 & 312 & 2212 & 63 & 9 & 27,0125 \\
\hline newill_d & 8 & 11 & 104 & 33 & 50 & 22 & 177 & 432 & 7 & 1 & 6,02758 & 51 & 401 & 3040 & 91 & 13 & 36,2447 & 23 & 177 & 884 & 70 & 10 & 16,5744 \\
\hline newtagd & 8 & 7 & 63 & 14 & 51 & 14 & 107 & 288 & 7 & 1 & 5,35582 & 24 & 192 & 900 & 77 & 11 & 18,9949 & 10 & 78 & 260 & 35 & 5 & 8,82142 \\
\hline rd84f1 & 8 & 8 & 99 & 21 & 57 & 120 & 1098 & 2196 & 7 & 1 & 32,4858 & 188 & 1418 & 37746 & 119 & 17 & 163,551 & 144 & 1082 & 26978 & 84 & 12 & 126,552 \\
\hline $\mathrm{rd} 84 \mathrm{f} 2$ & 8 & 7 & 63 & 15 & 57 & 128 & 1170 & 2340 & 7 & 1 & 28,5734 & 40 & 288 & 2892 & 56 & 8 & 30,3401 & 22 & 162 & 1028 & 42 & 6 & 14,4034 \\
\hline $\mathrm{rd} 84 \mathrm{f} 3$ & 8 & 7 & 62 & 14 & 56 & 1 & 27 & 54 & 7 & 1 & 3,52854 & 7 & 63 & 126 & 49 & 7 & 12,4683 & 7 & 63 & 294 & 21 & 3 & 7,68629 \\
\hline rd84f4 & 8 & 10 & 135 & 33 & 57 & 162 & 1476 & 2952 & 7 & 1 & 42,7301 & 176 & 1336 & 34468 & 119 & 17 & 158,735 & 142 & 1082 & 26504 & 84 & 12 & 130,351 \\
\hline $\max 46 \_d$ & 9 & 28 & 456 & 150 & 72 & 47 & 475 & 980 & 7 & 1 & 10,6472 & 185 & 1379 & 37264 & 112 & 16 & 210,26 & 156 & 1188 & 30628 & 98 & 14 & 141,284 \\
\hline sao2f1 & 10 & 9 & 187 & 46 & 73 & 10 & 124 & 264 & 7 & 1 & 5,7189 & 41 & 323 & 2320 & 91 & 13 & 27,696 & 39 & 309 & 2496 & 70 & 10 & 26,6478 \\
\hline sao2f 2 & 10 & 9 & 179 & 46 & 83 & 20 & 242 & 484 & 7 & 1 & 6,67963 & 60 & 474 & 5226 & 98 & 14 & 49,9699 & 45 & 345 & 3974 & 63 & 9 & 36,1185 \\
\hline sao2f3 & 10 & 9 & 149 & 34 & 71 & 92 & 914 & 2068 & 7 & 1 & 23,0123 & 130 & 1002 & 18000 & 126 & 18 & 105,365 & 80 & 628 & 9160 & 84 & 12 & 65,3744 \\
\hline sao2f4 & 10 & 9 & 159 & 32 & 69 & 85 & 898 & 1914 & 7 & 1 & 24,8451 & 137 & 1051 & 19684 & 133 & 19 & 111,094 & 83 & 651 & 9810 & 84 & 12 & 66,9386 \\
\hline sym10_d & 10 & 11 & 196 & 40 & 70 & 837 & 9229 & 18458 & 7 & 1 & 592,392 & 639 & 4721 & 372748 & 154 & 22 & 887,967 & 466 & 3494 & 240148 & 119 & 17 & 625,83 \\
\hline t481_d & 16 & 5 & 137 & 26 & 107 & 1547 & 21699 & 52666 & 7 & 1 & 2476,55 & 657 & 5033 & 292290 & 203 & 29 & 878,071 & 427 & 3253 & 177764 & 161 & 23 & 508,84 \\
\hline 5xp1 & 7 & 14 & 283 & 84 & 73 & 70 & $\begin{array}{l}385 \\
\end{array}$ & 2754 & 7 & 1 & 19,3511 & 140 & 985 & 23218 & $\overline{70}$ & 10 & 171,619 & $\overline{101}$ & 740 & 13030 & 63 & 9 & 86,1893 \\
\hline alu2 & 10 & 12 & 1030 & 284 & 148 & 257 & 2282 & 8448 & 7 & 1 & 106,399 & 404 & 3100 & 74522 & 280 & 40 & 4250,49 & 363 & 2767 & 71596 & 217 & 31 & 3192,4 \\
\hline alu4 & 14 & 8 & 3634 & 642 & 334 & 1791 & 19947 & 79200 & 7 & 1 & 4330,92 & 740 & 5706 & 199562 & 294 & 42 & 54801,5 & 657 & 5061 & 191338 & 238 & 34 & 39688,8 \\
\hline apex1 & 45 & 12 & 7975 & 1626 & 705 & 206 & 3018 & 44000 & 7 & 1 & 1471,98 & 2677 & 19430 & 5110198 & 189 & 27 & 11086,1 & 2020 & 14817 & 3827274 & 119 & 17 & 7804,18 \\
\hline apex2 & 39 & 10 & 1701 & 122 & 237 & 1035 & 15610 & 85198 & 7 & 1 & 1811,35 & 445 & 3627 & 134188 & 203 & 29 & 614,329 & 268 & 2134 & 71438 & 140 & 20 & 315,608 \\
\hline apex4 & 9 & 11 & 5727 & 2073 & 447 & 438 & 5489 & 24678 & 7 & 1 & 2875,89 & 3466 & 24824 & 9858816 & 147 & 21 & 18019,5 & 2744 & 19958 & 7830882 & 119 & 17 & 15452,3 \\
\hline apex5 & 117 & 16 & 6630 & 806 & 888 & 1160 & 8004 & 495908 & 7 & 1 & 1401,94 & 1293 & 9630 & 1239620 & 147 & 21 & 3189,77 & 832 & 6213 & 629036 & 84 & 12 & 1863,43 \\
\hline apex6 & 135 & 22 & 3761 & 770 & 1169 & 656 & 4926 & 353808 & 7 & 1 & 669,392 & 740 & 5138 & 569256 & 105 & 15 & 1405,3 & 674 & 4678 & 505306 & 98 & 14 & 1227,78 \\
\hline apex7 & 49 & 28 & 1937 & 290 & 437 & 507 & 4126 & 93740 & 7 & 1 & 315,733 & 252 & 1769 & 53172 & 98 & 14 & 279,106 & 197 & 1404 & 36774 & 91 & 13 & 232,827 \\
\hline b9 & 41 & 19 & 634 & 125 & 298 & 107 & 642 & 15996 & 7 & 1 & 73,3105 & 113 & 861 & 20768 & 70 & 10 & 117,437 & 92 & 690 & 15366 & 56 & 8 & 95,8065 \\
\hline clip & 9 & 21 & 485 & 120 & 89 & 166 & 1078 & 4816 & 7 & 1 & 41,0498 & 180 & 1354 & 33454 & 77 & 11 & 175,761 & 111 & 829 & 14098 & 63 & 9 & 107,43 \\
\hline $\mathrm{cm} 150 \mathrm{a}$ & 21 & 21 & 199 & 56 & 127 & 17 & 142 & 836 & 7 & 1 & 10,778 & 62 & 468 & 4502 & 91 & 13 & 50,1216 & 47 & 363 & 4102 & 63 & 9 & 48,2901 \\
\hline $\mathrm{cm} 162 \mathrm{a}$ & 14 & 14 & 198 & 46 & 102 & 43 & 230 & 1862 & 7 & 1 & 32,4292 & 38 & 298 & 2216 & 63 & 9 & 31,2726 & 33 & 257 & 1626 & 56 & 8 & 34,8671 \\
\hline $\mathrm{cm} 163 \mathrm{a}$ & 16 & 8 & 176 & 42 & 116 & 42 & 229 & 2016 & 7 & 1 & 23,054 & 36 & 288 & 2148 & 56 & 8 & 30,6175 & 32 & 254 & 1810 & 49 & 7 & 34,41 \\
\hline dalu & 75 & 13 & 4856 & 627 & 470 & 2224 & 26700 & 407862 & 7 & 1 & 5701 & 1387 & 10169 & 879430 & 245 & 35 & 20674,4 & 1122 & 8270 & 700910 & 217 & 31 & 15307,3 \\
\hline e64 & 65 & 24 & 840 & 94 & 456 & 65 & 2470 & 34060 & 7 & 1 & 125,161 & 1437 & 12134 & 416608 & 448 & 64 & 2436,47 & 520 & 3885 & 348982 & 70 & 10 & 1088,73 \\
\hline ex1010 & 10 & 11 & 6606 & 1984 & 396 & 1024 & 18950 & 41400 & 7 & 1 & 108896 & 3350 & 23952 & 8992430 & 168 & 24 & 17142,5 & 2616 & 19132 & 7027470 & 119 & 17 & 15397,7 \\
\hline ex4 & 128 & 7 & 3076 & 256 & 928 & 620 & 5220 & 124460 & 7 & 1 & 359,811 & 478 & 3672 & 299178 & 112 & 16 & 735,931 & 408 & 3124 & 248724 & 98 & 14 & 618,099 \\
\hline frg2 & 143 & 22 & 8433 & 803 & 1005 & 4159 & 35729 & 2424636 & 7 & 1 & 1351 & 1208 & 8712 & 1265816 & 91 & 13 & 3972,73 & 728 & 5142 & 518280 & 77 & 11 & 1768,91 \\
\hline misex1 & 8 & 18 & 231 & 83 & 69 & 18 & 132 & 780 & 7 & 1 & 7,30883 & 72 & 504 & 7314 & 49 & 7 & 59,2965 & 61 & 423 & 5740 & 42 & 6 & 46,7335 \\
\hline misex3 & 14 & 14 & 2969 & 444 & 185 & 1426 & 15559 & 80696 & 7 & 1 & 2430,17 & 1582 & 11604 & 1803240 & 161 & 23 & 4496,13 & 1167 & 8691 & 1280548 & 140 & 20 & 3152,18 \\
\hline misex3c & 14 & 10 & 2453 & 429 & 239 & 297 & 2710 & 18096 & 7 & 1 & 701,002 & 734 & 5380 & 483346 & 161 & 23 & 1188,36 & 590 & 4340 & 405166 & 126 & 18 & 1003,96 \\
\hline parity & 16 & 7 & 119 & 23 & 113 & 32768 & 557090 & 1114180 & 7 & 1 & $1,95466 \mathrm{e}+06$ & 46 & 346 & 3956 & 56 & 8 & 51,0531 & 46 & 346 & 3956 & 56 & 8 & 50,9398 \\
\hline $\mathrm{pdc}$ & 16 & 17 & 3658 & 507 & 142 & 2192 & 61318 & 250096 & 7 & 1 & $1,65169 \mathrm{e}+06$ & 1624 & 11994 & 1398822 & 182 & 26 & 4124,41 & 840 & 6290 & 627542 & 147 & 21 & 1741,17 \\
\hline seq & 41 & 11 & 10808 & 1566 & 692 & 1066 & 14502 & 167504 & 7 & 1 & 2323,22 & 2413 & 17877 & 3580692 & 203 & 29 & 9025,33 & 1775 & 13437 & 2541004 & 154 & 22 & 6382,24 \\
\hline squar5 & 5 & 18 & 224 & 93 & 56 & 30 & 261 & 1014 & 7 & 1 & 15,6276 & 68 & 484 & 6936 & 49 & 7 & 55,5393 & 48 & 326 & 3928 & 42 & 6 & 34,2392 \\
\hline $\mathrm{t} 481$ & 16 & 11 & 137 & 26 & 107 & 481 & 5267 & 16422 & 7 & 1 & 232,543 & 1875 & 13495 & 3463670 & 147 & 21 & 7784,26 & 803 & 5875 & 857962 & 91 & 13 & 2170,26 \\
\hline table5 & 17 & 14 & 4068 & 580 & 168 & 158 & 2566 & 11136 & 7 & 1 & 416,991 & 2001 & 14648 & 2370246 & 182 & 26 & 5730,48 & 1481 & 11082 & 1841246 & 154 & 22 & 4415,77 \\
\hline tcon & 17 & 7 & 166 & 57 & 118 & 32 & 154 & 3234 & 7 & 1 & & 48 & 298 & 6034 & 21 & 3 & 35,5259 & 40 & 226 & 5218 & 14 & 2 & 29,4298 \\
\hline term1 & 34 & 10 & 896 & 105 & 260 & 257 & 2298 & 23584 & 7 & 1 & 88,2616 & 313 & 2420 & 107300 & 112 & 16 & 393,415 & 150 & 1165 & 29526 & 84 & 12 & 162,668 \\
\hline too_large & 38 & 14 & 2866 & 282 & 232 & 1035 & 15610 & 85198 & 7 & 1 & 1389,22 & 827 & 6375 & 411848 & 210 & 30 & 1394,65 & 463 & 3621 & 194054 & 168 & 24 & 699,077 \\
\hline $\mathrm{vda}$ & 17 & 11 & 3039 & 805 & 273 & 93 & 1544 & 14896 & 7 & 1 & 376,691 & 955 & 6855 & 640324 & 112 & 16 & 2693,42 & 650 & 4638 & 444516 & 70 & 10 & 1737,85 \\
\hline $\mathrm{x} 1$ & 51 & 11 & 2810 & 230 & 398 & 309 & 2624 & 59340 & 7 & 1 & 156,096 & 400 & 3007 & 179366 & 91 & 13 & 544,872 & 286 & 2091 & 123350 & 70 & 10 & 371,451 \\
\hline $\mathrm{x} 2$ & 10 & 11 & 206 & 60 & 80 & 17 & 118 & 850 & 7 & 1 & 7,6228 & 60 & 428 & 5680 & 49 & 7 & 52,4899 & 46 & 314 & 3636 & 42 & 6 & 42,7509 \\
\hline $\mathrm{x} 3$ & 135 & 22 & 3761 & 770 & 1169 & 738 & 5535 & 392184 & 7 & 1 & 648,998 & 841 & 6241 & 893392 & 105 & 15 & 1961,42 & 611 & 4527 & 550626 & 77 & 11 & 1221,77 \\
\hline $\mathrm{x} 4$ & 94 & 39 & 3042 & 401 & 642 & 530 & 3431 & 198660 & 7 & 1 & 329,832 & 470 & 3378 & 207320 & 70 & 10 & 660,801 & 341 & 2403 & 112264 & 63 & 9 & 406,664 \\
\hline
\end{tabular}

COMPARISON OF BENCHMARKS

\section{A. Experiments}

In order to investigate about the impact of different synthesis optimization parameters on the memristor crossbar in terms of area and performances, we carried out several experiments.

Given a function expressed in BLIF format, we first used synthesis tools (i.e. ABC and SIS by Berkeley) in order to produce EQN format. Specifically we used:

- SIS to generate equations in which outputs depend directly on inputs, so they can be translated to a single crossbar

- $\mathrm{ABC}$ to generate equations in which outputs depend on intermediate values, so they can be translated to more crossbars. Also an "optimized" version has been produced using the resyn 2 script [15]

An overview about achieved results is presented and discussed hereafter. In table II a legend of the used labels is reported.

\section{B. Discussion}

Table I shows the synthesis results on a number of combinational benchmarks. We noticed that:

1) As the levels' number of a function grows - thus the number of crossbars and, hence, the latency - the number of memristor grows instead of decreasing.

2) Given the number of crossbars, the response time of the circuit remains constant for any function.

a) Therefore, in terms of both area and performances, is better to have only a single crossbar in order to obtain a tiny and constant time and use fewer memristors.

3) The number of minterms has an high impact on the memristors' total number.

a) At the current state of art, there is not any tool that makes possible to optimize a boolean function in terms of number of minterms. Maybe the next direction could be such. 


\begin{tabular}{|c|c|c|c|}
\hline Symbol & Description & \multicolumn{2}{|c|}{ Expressed as } \\
\hline$p_{i j}$ & Whether the $i$-th minterm is in the $j$-th level (boolean) & \multicolumn{2}{|l|}{ Given } \\
\hline$N_{i n}\left(l_{j}\right)$ & Num. of input for the $j$-th level & \multicolumn{2}{|l|}{ Given } \\
\hline$N_{\text {out }}\left(l_{j}\right)$ & Num. of output for the $j$-th level & \multicolumn{2}{|l|}{ Given } \\
\hline$N_{o c c}\left(m_{i}, l_{j}\right)$ & Num. of occurrences of the $i$-th minterm in the $j$-th level & \multicolumn{2}{|l|}{ Given } \\
\hline$N_{l i t}\left(m_{i}\right)$ & Num. of literals of the $i$-th minterm & \multicolumn{2}{|c|}{ Given } \\
\hline$N_{m e m}$ & No. of memristors in the circuit & \multicolumn{2}{|c|}{$\left[2 * N_{\text {in }}\left(l_{j}\right)+\sum_{i}\left(N_{\text {occ }}\left(m_{i}, l_{j}\right)\right)+\sum_{i}\left(N_{\text {lit }}\left(m_{i}\right) * p_{i j}\right)+2 * N_{\text {out }}\left(l_{j}\right)\right.$} \\
\hline$N_{\min }$ & No. of minterms in the circuit & \multicolumn{2}{|c|}{ Given } \\
\hline A & Total area & $\sum_{j}\left\{\left[2 * N_{\text {in }}\left(l_{j}\right)+2 * N_{\text {out }}\left(l_{j}\right)\right] *\right.$ & 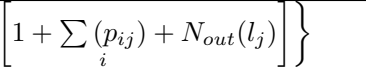 \\
\hline$t_{M}$ & Memristor switching time & \multicolumn{2}{|c|}{ Given } \\
\hline$N_{\text {Steps }}$ & Num. of steps of a Crossbar computation & \multicolumn{2}{|c|}{ Given } \\
\hline$T_{C}$ & "Latency" of a Crossbar & \multicolumn{2}{|c|}{$t_{M} * N_{\text {Steps }}$} \\
\hline$N_{C}$ & Num. of Crossbar in the circuit & \multicolumn{2}{|c|}{ Given } \\
\hline $\mathrm{C}$ & Needed Cycles to complete computation & \multicolumn{2}{|c|}{$T_{C} * N_{C}$} \\
\hline$T_{X b G}$ & Time (in milliseconds) needed by XbarGen to execute & \multicolumn{2}{|c|}{ Experimental } \\
\hline$T_{\text {sim }}$ & Time (in milliseconds) for simulate VHDL design (50ns long) & \multicolumn{2}{|c|}{ Experimental } \\
\hline \multicolumn{4}{|c|}{ Chakraborti et al. [7] } \\
\hline$M_{s}$ & \multicolumn{3}{|l|}{ No. of memristors (serial) } \\
\hline$O P_{s}$ & \multicolumn{3}{|l|}{ No. of memristor micro-operations (serial) } \\
\hline$M_{p}$ & \multicolumn{3}{|l|}{ No. of memristors (parallel) } \\
\hline$O P_{p}$ & \multicolumn{3}{|l|}{ No. of memristor micro-operations (parallel) } \\
\hline
\end{tabular}

In addition, with regard to feasability of translation and simulation of memristor crossbar behavioral circuits, we collected the time that XbarGen needs to translate a boolean function and produce the crossbar schematic view (table I). XbarGen experiments were run on a dual-core i7 MacBook Pro with 2,8GHz clock, 4 GB RAM and running OSX v10.11.4.

\section{Comparison}

Chakraborti et al. in [7] proposed an architecture based on material implication operation implemented using memristors. In brief, they came up with a realization of 2-to-1 multiplexer using memristors, and a synthesis methodology that represents a given Boolean function as a Reduced Ordered Binary Decision Diagram (ROBDD) and maps it to memristor implementation. They carried out some benchmarks too (table I), reporting interesting results: comparing experiments that exploit parallelism and those that do not, the inverse ratio time-area is respected; from our side, instead, it is not true. On the other hand, execution time of the architecture in [7] depends on the function under consideration; execution time of Snider architecture could be independent from the function, if it is translated to a single crossbar. This could mean that one would prefer an architecture such as proposed in [7] in case of area constraints and an architecture such as Snider proposed in [8] in case of time constraints.

\section{CONCLusion}

The memristor is one of the most promising technologies which is able to deal with the CMOS limitations. In particular, since the memristor is inherently able to behave also as a non-volatile device, it allows to overcome the bottleneck of the data transfer to the computational unit and back to storage elements. The research community is facing with the synthesis of boolean functions by exploiting memristor-based crossbars and, in this paper, we advanced the state-of-the-art with a comparison with the state-of-the-art of performances w.r.t. several benchmarks. Indeed, we developed XbarGen, a tool which is able to process boolean equations for the mapping over memristor crossbars.

\section{REFERENCES}

[1] ITRS 2013 report. [Online]. Available: http://www.itrs.net/

[2] B. Hoefflinger, "Chips 2020: A Guide to the Future of Nanoelectronics", The Frontiers Collection, Springer Berlin Heidelberg, 2012, pp. 421427

[3] J. McPherson, "Reliability trends with advanced CMOS scaling and the implications for design", in IEEE Custom Integrated Circuits Conference, 2007 , pp. 405-412

[4] S. Borkar, "Design perspectives on 22Nm CMOS and beyond", in Proceedings of the 46th Annual Design Automation Conference, 2009, pp. 93-94

[5] G. Gielen, et al., "Emerging yield and reliability challenges in nanometer CMOS technologies", in Proceedings of the Conference on Design, Automation and Test in Europe, pp. 1322-1327, 2008

[6] S. Hamdioui, et al., 'Memristor based computation-in-memory architecture for data-intensive applications', in Proceedings of the Conference on Design, Automation and Test in Europe, pp. 1718-1725, 2015

[7] S. Chakraborti, P. V. Chowdhary, K. Datta and I. Sengupta, "BDD based synthesis of Boolean functions using memristors," 2014 9th International Design and Test Symposium (IDT), Algiers, 2014, pp. 136-141.

[8] G. Snider, "Computing with hysteretic resistor crossbars," Applied Physics A, vol. 80, pp. 1165-1172, 2005.

[9] L. Chua, 'Memristor-the missing circuit element', IEEE Transactions on Circuit Theory, vol. 18, no. 5, pp. 507-519, 1971

[10] R. Waser et al., "Redox-based resistive switching memories-nanoionic mechanisms, prospects, and challenges," Advanced Materials, vol. 21, pp. 2632-2663, 2009

[11] J. J. Yang et al., "Memristive devices for computing," Nature nanotechnology, vol. 8, pp. 13-24, 2013

[12] J. R. Burger et al., "Variation-tolerant computing with memristive reservoirs," IEEE/ACM International Symposium in Nanoscale Architectures (NANOARCH), 2013, pp. 1-6.

[13] K.-H. Kim et al., "A functional hybrid memristor crossbar-array/cmos system for data storage and neuromorphic applications," Nano letters, vol. 12, pp. 389-395, 2011. 
[14] Lei Xie, Hoang Anh Du Nguyen, Mottaqiallah Taouil, Said Hamdioui, Koen Bertels, "Fast Boolean Logic Mapped on Memristor Crossbar", IEEE International Conference on Computer Design (ICCD), pp. 335$342,2015$.

[15] ABC User Guide. [Online]. Available: http://www.eecs.berkeley.edu/ alanmi/abc/ 